\title{
婴儿游泳护理教育的 PDCA 试验
}

\section{PDCA Test of Infant Swimming Nursing Education}

\section{朱艳芬}

Yanfen Zhu

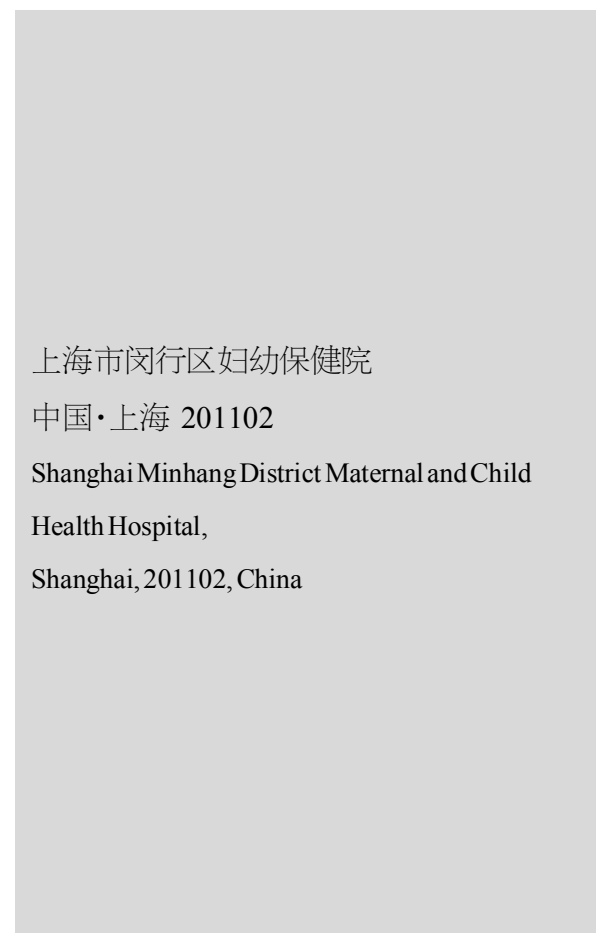

【摘要】论文首先通过对婴儿游泳护理实践活动进行内涵界定,其次进行现状分析。得出 了婴儿游泳护理的过程管理, 是摆在广大护理工作者面前亟待解决的现实问题, 由此提出 护理教育 PDCA 模式。通过对 PDCA 教育模式架构分析、实践试验分析、有效性分析等, 提 出护理教育 PDCA 模式的改进措施。最后得出, 婴儿游泳护理 PDCA 模式是三论原理融入 医院管理的又一次运用,是提高医院婴儿护理、促进护理教育良性循环的重要保障。

【Abstract】Firstly, this paper defines the connotation of infant swimming nursing practice, and then carries on the analysis of the current situation. It is concluded that the process management of infant swimming nursing is a practical problem to be solved urgently in front of the vast number of nursing workers, and the PDCA model of nursing education is put forward accordingly. Through the analysis of PDCA education model structure, practice experiment analysis and effectiveness analysis, the improvement measures of PDCA model in nursing education are put forward. Finally, it is concluded that PDCA model of nursing education is another application of enterprise management into hospital management; it is an important guarantee to improve the level of hospital nursing management and promote the virtuous cycle of nursing education activities.

【关键词】婴儿游泳; PDCA 模式; 过程管理; 有效性分析

【Keywords 】baby swimming; PDCA model; process management; effective analysis 【DOI】10.36012/pmr.v1i1.156

\section{1 现状分析}

贞儿游泳是指贞儿在经过专门培训的护理人员操作和监 护下进行的一项全新的贞儿保健教育活动, 是把水当载体, 把 婴儿自主活动、被动抚触相结合, 可反射性引起婴儿全身反 应, 直接刺激婴儿的视、嗅、触觉, 间接地作用到婴儿身体各个 系统, 促进㚣儿身心健康发育, 有利于贞儿早期智力发育的一 项教育实践。

然而, 今天的妇幼保健医院多数贞儿游泳没有预案, 过程 管理不够到位、方法不够创新, 事后总结反馈没有等一系列问 题。随着贞儿护理的深入发展, 创新贞儿护理过程性管理, 是 摆在护理工作者面前亟待解决的现实问题。

\section{2 理论依据}

“三论”即信息论、系统论和控制论的简称, 三论原理是 20 世纪人类管理科学研究的重大突破。三论原理揭示的是有机 界与无机界的联系和规律, 它促进了管理向综合化、整体化方
向发展, 比如,物联网就是三论的应用 ${ }^{[1,2]}$ 。三论原理同时对探 讨管理领域的实践问题提供了有力的理论支持。其中的系统 论对组织管理、过程管理等都有特别的指导意义,提出了管理 系统的指导理论与方法。另外,任何的管理过程都是一个信息 控制的过程，三论中的信息论、控制论对管理的监理信息系 统、控制系统、提高管理的控制能力、管理的过程环节等都具 有重要的指导意义。美国戴明博士提出的 PDCA 过程管理就 是控制管理过程的一种很有效的方法，也是三论原理的实践 版,对婴儿护理有很好的指导。

\section{PDCA 模式架构}

人们已经知道，良好的过程管理是达成管理目标的重要 保障, 所谓的过程其实就是一组将实践活动“输入”转化为学 生素质 “输出” 的相互关联或相互作用的一系列的教育过程。 在贞儿游泳护理教育活动中, 人们希望通过游泳活动的 “输 人”, 获得婴儿身心发育, 早期智力提升的“输出”。笔者医院在 婴儿护理活动的管理中, 积极融合了过程性管理思想, 借用了 
PDCA 模式进行活动的过程性控制[13,4]。PDCA 由美国质量管理 专家戴明首先提出, 为动态循环的过程管理模式。实践中 PDCA 主要包括: 婴儿游泳护理教育活动计划 $(\mathrm{P})$ 、婴儿游泳护 理教育执行 $(D)$ 、婴儿游泳护理教育检查 $(C)$ 和贞儿游泳护理 教育反思改进 $(\mathrm{A})$ 等。在实践操作中一般 $\mathrm{P}$ 阶段为活动的计划 方案、目标、预期效果等; $\mathrm{D}$ 为执行阶段, 就是实现活动计划, 过程与细节等; $\mathrm{C}$ 阶段为检查、总结执行的结果情况, 找出执 行中出现的问题, 预期效果的检测等; A 为行动改进阶段, 也 是 PDCA 总结检查的处理阶段, 此阶段要把成功的经验给以 肯定，把活动流程标准化、流程进行再造，从而给予下一个 PDCA 护理实践来推广。这样的一个过程也就是形成了具有护 理教育特色的实践活动管理模式即护理活动的 “设计方案一 组织实施一检查反馈一反思改进”的过程管理。

贞儿游泳护理教育实践证明, PDCA 模式是提高贞儿游 泳护理教育过程管理的有效手段，可以大幅提升护理实践的 有效性。

\section{4 实践试验}

婴儿游泳护理教育融入 PDCA 模式后，实施路径与方法 如下。

\section{1 婴儿游泳护理教育活动计划( $P$ )}

$\mathrm{P}$ 阶段就是根据护理实践活动的方案要求、组织方针, 为 提供㚣儿游泳建立必要的预案、目标和预期效果等。首先对贞 儿游泳护理教育进行调研, 论证其可行性与必要性,避免造成 人力、物力的大量浪费; 紧接分层提出活动方案, 用来适应不 同婴儿的需求,節选确定最佳活动方案; 最后就是制定婴儿游 泳护理教育相应对策、制定护理计划。

\section{2 婴儿游泳护理教育执行 (D)}

有了方案、计划, 接下来就是 $\mathrm{D}$, 即按照活动预定, 分工内 容去执行与实施了, 这就是实现护理预期方案的目标过程。实 践中人们在护理婴儿游泳的同时还搜集和建立了相关数据采 集,收集实施过程中原始数据和记录等, 为 PDCA 后期的有效 性分析做准备。游泳前准备: 首先做好宣教, 告知家属㚣儿游 泳目的、好处等, 以取得密切配合。执行游泳消毒隔离: 严格消 毒隔离, 一贞一巾一换。㚣儿游泳完, 要清洗泳池, 消毒泳圈, 用紫外线消毒泳室空气,以备后用。在此阶段最重要的护理就 是安全。婴儿游泳前要仔细检查游泳圈安全性,套游泳圈后要 检查㚣儿下领部位是否垫托在预设位置, 扣好双重保险装置, 粘贴好防水脐贴后将婴儿缓慢放入水中自由游泳。

\section{3 贞儿游泳护理教育检查 (C)}

也就是检查贞儿游泳活动过程的效果，是否达到预期效
果。实践中是通过贞儿游泳教育活动分工、活动记录、总结报 告等项目来对婴儿游泳活动效果评估的。一般护理人员在贞 儿游泳结束后, 要迅速擦干婴儿身上的水迹, 并且要注意保 暖,取下防水护脐贴, 常规进行脐部护理 ${ }^{[5]}$

\section{4 婴儿游泳护理教育反思改进 $(A)$}

贞儿游泳教育活动结束后, 要总结反思, 处理本次护理教 育中遗留的问题, 这个阶段叫作反思改进(Action)。此阶段要 把成功的经验给以肯定, 把贞儿游泳护理活动流程标准化、流 程进行再造, 从而给予下一个 PDCA 实践来推广应用。对于贞 儿游泳活动方案实施过程中出现的问题或者实施效果不显著 的, 要进行修正、总结, 这也为下新一轮婴儿游泳活动 PDCA 循环提供新依据。

\section{5 有效性分析}

这种“婴儿游泳活动 PDCA 模式”的效果到底如何即有效 性怎样呢?

笔者设计了下表 1 “PDCA 护理教育活动家属认可度”调 查问卷。

表 1 婴儿游泳护理 PDCA 试验护理人员反映

\begin{tabular}{c|c|c}
\hline 题号 & 调研问题 & $\begin{array}{c}\text { 愿意打几分 } \\
(0 \sim 7 \text { 分 })\end{array}$ \\
\hline 1 & 婴儿游泳护理 PDCA 活动从婴儿发展需求制定 & \\
\hline 2 & 婴儿参与游泳程度 & \\
\hline 3 & 这一模式下能快速提升婴儿身体素质 & \\
\hline 4 & 这种过程性管理法对活动的有效监控 & \\
\hline 5 & PDCA 过程管理培养护理人员职业素养 & \\
\hline 6 & 婴儿游泳护理 PDCA 实践活动的有效性 & \\
\hline 7 & 希望今后推广婴儿游泳护理 PDCA 管理 & \\
\hline
\end{tabular}

本院随机抽取 50 家属, 3 人问卷由于填报不完整作废, 最 后确定有效问卷 47 份。进行数理分析得到表 2 。

表 2 分析结果

\begin{tabular}{c|c|c|c|c|c}
\hline 题号 & 总分 & 最低分 & 最高分 & 平均值 & 标准差 \\
\hline 1 & 279 & 5 & 7 & 5.9362 & 0.6966 \\
\hline 2 & 242 & 4 & 7 & 5.1489 & 0.8500 \\
\hline 3 & 232 & 3 & 7 & 4.9362 & 1.1186 \\
\hline 4 & 240 & 3 & 7 & 5.1064 & 1.2245 \\
\hline 5 & 239 & 3 & 7 & 5.0851 & 1.1998 \\
\hline 6 & 242 & 3 & 7 & 5.1489 & 1.3043 \\
\hline 7 & 268 & 4 & 7 & 5.7021 & 0.8972 \\
\hline
\end{tabular}

对数据进行统计作图, 可以得到图 1。

根据表 2、图 1 的分析,可以看出:

(1)护理工作者对于护理 PDCA 护理过程管理基本保持肯 定态度, 每项的平均分都在 5 分以上。实践让护理人员在诸如 
PDCA 实践活动护理人员反映

$\rightarrow$ 最低分 - - 平均值 $\multimap$ 标准差

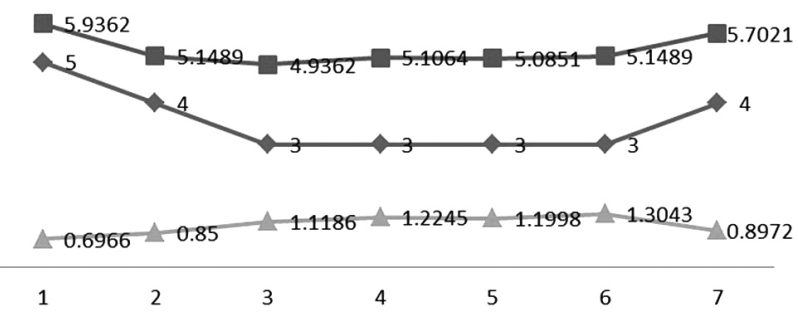

图 1 问卷分析

职业素养、专业能力、集体意识、职业态度等方面都有收获,标 准差值都较小, 不超过 1.5 , 数据说明被调查人员意见相对一 致。

(2)调研第 6 问: 婴儿游泳护理 PDCA 实践活动的有效性, 护理人员认可度平均得分 6 分, 从这个数据人们可以看出, 护 理人员对于婴儿游泳 PDCA 效果持高度肯定。

(3)下面再看护理人员认可度的最低分统计, 从上表数据 看没有给出低于 3 分的, 这个数据表明, 护理人员对 PDCA 实 践没有抵触情绪, 根据活动后期调查、访谈、分析, 得到的结论 是:参与的护理人员均表示希望今后推广婴儿游泳护理 PDCA
管理。

\section{6 结论}

通过多年的贞儿游泳护理 PDCA 管理, 总结了以下几点: 第一,护理实践活动应作为护理教育常设课程。形成的“护理 设计方案一护理组织实施一护理检查指导一护理总结改进” 的 PDCA 过程管理应该作为护理项目课程常抓不解。第二,建 议在贞儿游泳护理 PDCA 实践前, 做好 PDCA 计划预设, 针对 婴儿身心, 做好参与 PDCA 人员的辅导。

总之，婴儿游泳护理 PDCA 是三论原理融入医院管理的 再利用, 是提高护理管理水平的又一种路径。人们将继续在护 理的实践中探索 PDCA 过程管理的有效性,争取做实做好。

\section{参考文献:}

[1](日)川原慎也.PDCA 精进法[M].谌红军,译.北京:东方出版社, 2017.

[2]王文博,牛雪䑣.看图学游泳[M].北京: 人民邮电出版社,2015.

[3]谢宗豹,林惠青. 医学思维与创新 $[\mathrm{M}]$.上海:科学技术出版社 2009.

[4]余剑珍.护理管理学基础[M].北京:科学出版社,2003.

[5]马斌荣.医学统计学 $[\mathrm{M}]$. 北京: 人民卫生出版社,1990. 